\title{
Intragenesis as a Sustainable Crop Improvement Method: A Review
}

\author{
Mainak Barman ${ }^{1}$, Saipayan Ghosh ${ }^{2 *}$, Kingsuk Das ${ }^{3}$ and Sabir Ahmed Mondol ${ }^{4}$ \\ ${ }^{1}$ Department of Plant Breeding and Genetics, Dr. Rajendra Prasad \\ Central Agricultural University, Pusa, Bihar, India \\ ${ }^{2}$ Department of Agricultural Biotechnology and Molecular Biology, \\ Dr. Rajendra Prasad Central Agricultural University, Pusa, Bihar, India \\ ${ }^{3}$ Department of Agricultural Biotechnology, \\ Assam Agricultural University, Jorhat, Assam, India \\ ${ }^{4}$ Department of Biochemistry, Uttar Banga Krishi Viswavidyalaya, West Bengal, India \\ *Corresponding author
}

\section{A B S T R A C T}

Keywords

Genetically

modified

organisms,

Intragenic

modification,

Sexually

compatible gene

pool

Article Info

Accepted:

07 March 2020

Available Online:

10 April 2020
Our society has a great concern about transgenic crops regarding mixing of genetic materials between species whose hybridization is not possible naturally. Different public policies on the development and use of genetically modified organisms (GMOs) are of high concern with defining proper risk management strategies. Researchers are in agreement that this technology "tampers with nature" in an unacceptable manner. This has been recognised as an objection to the crossing of species borders in generation of transgenic organisms. There are recent projects of genetic modification which is aimed at accommodation of the above mentioned concerns by alteration of expression of endogenous genes rather than introduction of genes from other species. Several surveys show higher public acceptance of intragenic/cisgenic crops compared to transgenic crops. Therefore, although the intragenic and cisgenic concepts were internationally introduced only in last decade, several different traits in different variety of crops have recently been modified according to these concepts. The main moral reason for intragenic modification, is the requirement to respect the "otherness" of nature.

\section{Introduction}

The area of agricultural land utilized for the production purpose of transgenic (genetically modified or GM) crops has augmented at an unprecedented swiftness as the journey of these crops were commenced about 22 years ago. Even though the introduced Genetically Modified crops have been exceedingly triumphant, it is evident that only a part of the 
potential of genetic modification of crop plants is being grasped (Holme et al., 2013).

The technological as well as economical inconveniences are, however, only a fraction of the dilemma. Worldwide, the GMtechnology has been met considerable scepticism among the common people and in effect thereof by the producers also, the industry along with the retailers.

Various studies evidently demonstrate that one of the chief anxieties of the public about transgenic crops is the combination of genetic elements artificially derived from diverse organisms that are not crossable by the natural means (Gaskell and Bauer, 2001; Bauer and Gaskell, 2002; Lassen et al., 2002).

This reluctance is linked time and again to a kind of respect for mother nature and also comes into view to be inter-linked with worries for impending health jeopardies and for the scattering of novel gene-combinations into the environment.

Bearing public worries about safety issues in mind concerning the GM crops in addition to the aspire of meeting these doubts and at the same time certifying an environmentally sound and resourceful plant production, the concept of intragenesis was developed as an efficient substitute to the transgenic crop development (Jacobsen and Schouten, 2007).

The concept is based on the elite utilization of genetic material from the same species or genetic material from the species that are closely-related and able to perform sexual hybridization.

This is in distinguishing to transgenesis where the genes as well as DNA sequences can be shifted between any species (Rommens et al., Jacobsen and Schouten, 2007).

\section{Issues associated with traditional plant} breeding

\section{Unintentional transfer of undesirable genes}

In their hard works to have room for the evolving requirements of growers as well as consumers, plant breeders take up any accessible tool to recognize the strongest feasible traits. In one application, a quantity of the genetic diversity that is obtainable within a sexually compatible group is captured by making crosses between the cultivated varieties and the wild relatives and screening the consequential $F_{1}$ hybrids for a novel trait. Through performing extensive backcrossing, a part of the wild DNA, representing at least $\sim 1 \%$ of the whole genome and encompassing the selected trait, is introgressed into the existing varieties.

Significantly, the DNA segment that is introgressed may include one or numerous genes that are linked with detrimental characteristics. Over and above frequently observed linkage yield drag (Kopisch-Obuch et al., 2005), introgression can consequence in ambiguous alterations linked to lower quality of food. For example, transfer of "crisp chip" and "high starch" traits from Solanum chacoense to cultivated potato (Solanum tuberosum) augmented the levels of glycoalkaloid in the resultant variety Lenape to approximately twice concentration of the highest allowed limit $\left(354 \mu \mathrm{g} \mathrm{kg}^{-1}\right.$ ) (Akeley et al., 1968; Zitnack et al., 1970).

\section{Persistent incidence of plant-produced toxins or allergens}

More than $99 \%$ of the total dietary intake of toxins has been estimated to be generated by the food crops themselves (Ames et al., 1990). Products, for example, acrylamide, exhibit properties of being toxic as well as carcinogenic and gather in, such as, bread 
crusts along with the surface of potato chips in addition to French fries (Tareke et al., 2002). The normal acrylamide dietary intake level is $28 \mu \mathrm{g} \mathrm{day}{ }^{-1}$ (www.cfsan.fda.gov/ dms/acryexpo.html.) and it could be lessen to slight quantities by the use of wheat and potato varieties accumulating stumpy levels of asparagine.

Unfortunately, breeders have not yet selected for low asparagine, and there are no acceptable varieties available at present that unexpectedly demonstrate this trait. Thus, labours to produce low-asparagine potato or wheat would necessitate both identification of source and introgression of trait, a procedure that may take a long time of 20 years (Rommens, 2007).

The availability of genes in crops of these days that are allergen-encoding is an even more vital concern. A solitary peanut can be menacing to people's life liable to budding allergy reactions, and the consumption of bread often injures the intestinal lining of $0.8 \%$ of the Americans unintentionally that suffer from gluten sensitivities (Harrison et al., 2007).

Although a number of allergen-encoding genes have been inactivated through the use of mutagenesis, it would be complicated to get rid of all 20-80 genes from the crops such as rice, wheat, soybean, peanut, and apple that encode allergens or suspected allergens (Gendel and Jenkins, 2006).

The regulatory agencies resist the deliberate utilization of genes that are identified to generate toxins, allergens, or anti-nutritionals (Kaeppler, 2000) but can accomplish petite to stop the unchecked transfer of such genes through conventional breeding (Bradford et $a l ., 2005)$. There is another major issue of dormant traits activation.

\section{Intragenesis}

Rommens introduced the definition of the concept of intragenic transformation in the year 2004. In intragenesis, diverse plant genetic components are recombined in-vitro to raise an expression gene construct that is then introduced into a plant falling within the same sexually compatible gene pool (Rommens 2004; Rommens et al., 2007).

Thus, the source of the genes for the genetic modification in case of intragenesis is from the same or a species that falls within crossable limit. Intragenes are hybrid genes. Selected genetic elements from various genes can be merged together in intragenesis (Rommens et al., 2007). Intragenesis permits for the design of cassettes uniting definite genetic elements from plants belonging to the identical sexually compatibile gene pool. So, coding regions of one gene (with or without the introns) can be combined with the promoters and the terminators from various genes from the same sexually compatibile gene pool.

Moreover, silencing constructs can be designed by merging a number of different genetic elements from the group having the same sexual compatibility. When employing Agrobacterium-mediated transformation, the T-DNA border sequences should derive from the sexually compatible DNA pool (P-DNA borders). The phenotype of a plant achieved through the intragenesis is not attainable through the approaches of traditional breeding as the expression profile of the freshly accumulated gene may vary from that detected naturally (Schaart and Visser 2009; Devi et al., 2013). Antisense or RNA interference (RNAi) can be utilized for achieving the purpose of silencing the gene(s) in case of intragenesis (Schaart and Visser 2009) (Table 1 and 2). 
Table.1 Examples of traits that can be incorporated into a plant by either transferring or modifying the expression of native genes

\begin{tabular}{|c|c|c|c|c|c|}
\hline Trait & target plant & target gene & approach & Year & Reference \\
\hline $\begin{array}{l}\text { Extended shelf } \\
\text { life }\end{array}$ & tomato & $\mathrm{Pg}$ & $\begin{array}{l}\text { fruit-specific } \\
\text { silencing }\end{array}$ & 1988 & $\begin{array}{l}\text { Sheehy et } \\
\text { al., } 1988\end{array}$ \\
\hline $\begin{array}{l}\text { Extended shelf } \\
\text { life }\end{array}$ & tomato & Acc oxidase & $\begin{array}{l}\text { fruit-specific } \\
\text { silencing }\end{array}$ & 1991 & $\begin{array}{l}\text { Oeller et al., } \\
1991\end{array}$ \\
\hline $\begin{array}{l}\text { Extended shelf } \\
\text { life }\end{array}$ & tomato & $\begin{array}{l}\text { Acc } \\
\text { synthase }\end{array}$ & $\begin{array}{l}\text { fruit-specific } \\
\text { silencing }\end{array}$ & 1998 & $\begin{array}{l}\text { Liu et al., } \\
1998\end{array}$ \\
\hline $\begin{array}{l}\text { Increased } \\
\text { vitamin-E } \\
\text { content }\end{array}$ & Arabidopsis & gmt & $\begin{array}{l}\text { seed-specific } \\
\text { overexpression }\end{array}$ & 1998 & $\begin{array}{l}\text { Shintani et } \\
\text { al., } 1998\end{array}$ \\
\hline $\begin{array}{l}\text { Reduced } \\
\text { glycemic index }\end{array}$ & potato & $\begin{array}{l}\text { Sbe I } \\
+ \\
\text { Sbe II }\end{array}$ & $\begin{array}{l}\text { tuber-specific } \\
\text { silencing }\end{array}$ & 2000 & $\begin{array}{l}\text { Schwall et } \\
\text { al., } 2000\end{array}$ \\
\hline $\begin{array}{l}\text { Increased } \\
\text { xanthophyll } \\
\text { content }\end{array}$ & tomato & $\begin{array}{l}\text { Lcy } \\
+ \\
\text { Chy }\end{array}$ & $\begin{array}{l}\text { fruit-specific } \\
\text { overexpression }\end{array}$ & 2002 & $\begin{array}{l}\text { Dharmapuri } \\
\text { et al., } 2002\end{array}$ \\
\hline $\begin{array}{l}\text { Heat-stable } \\
\text { vegetable oil }\end{array}$ & cottonseed & Fad2 & $\begin{array}{l}\text { seed-specific } \\
\text { silencing }\end{array}$ & 2002 & $\begin{array}{l}\text { Liu et al., } \\
2002\end{array}$ \\
\hline $\begin{array}{l}\text { Increased } \\
\text { zeaxanthin } \\
\text { content }\end{array}$ & potato & Zep & $\begin{array}{l}\text { tuber-specific } \\
\text { silencing }\end{array}$ & 2002 & $\begin{array}{l}\text { Romer } \\
\text { al., } 2002\end{array}$ \\
\hline $\begin{array}{l}\text { Increased } \\
\text { anthocyanin } \\
\text { content }\end{array}$ & tomato & Ant1 & $\begin{array}{l}\text { fruit-specific } \\
\text { overexpression }\end{array}$ & 2003 & $\begin{array}{l}\text { Mathews et } \\
\text { al., } 2003\end{array}$ \\
\hline $\begin{array}{l}\text { Increased } \\
\text { vitamin-C } \\
\text { content }\end{array}$ & strawberry $^{\mathrm{a}}$ & GalUR & $\begin{array}{l}\text { constitutive } \\
\text { overexpression }^{\mathrm{b}}\end{array}$ & 2003 & $\begin{array}{l}\text { Agius et al., } \\
2003\end{array}$ \\
\hline $\begin{array}{l}\text { Increased } \\
\text { vitamin-E } \\
\text { content }\end{array}$ & soybean & $\begin{array}{l}\text { Vte3 } \\
+ \\
\text { Vte4 }\end{array}$ & $\begin{array}{l}\text { seed-specific } \\
\text { overexpression }^{c}\end{array}$ & 2003 & $\begin{array}{l}\text { Van- } \\
\text { Eenennaam } \\
\text { et al., } 2003\end{array}$ \\
\hline $\begin{array}{l}\text { Late blight } \\
\text { resistance }\end{array}$ & potato & $\mathrm{RB}$ & $\begin{array}{l}\text { use of original } \\
\text { promoter }\end{array}$ & 2003 & $\begin{array}{l}\text { Song et al., } \\
2003\end{array}$ \\
\hline $\begin{array}{l}\text { Reduced } \\
\text { allergen } \\
\text { content }\end{array}$ & soybean & $\begin{array}{l}\text { Gly } \mathrm{m} \mathrm{Bd} \\
30 \mathrm{~K}\end{array}$ & $\begin{array}{l}\text { constitutive } \\
\text { silencing }^{\mathrm{d}}\end{array}$ & 2003 & $\begin{array}{l}\text { Herman } \\
\text { al., } 2003\end{array}$ \\
\hline $\begin{array}{l}\text { Enhanced } \\
\text { aroma }\end{array}$ & potato & Cgsb & $\begin{array}{l}\text { constitutive } \\
\text { overexpression }^{\mathrm{b}}\end{array}$ & 2003 & $\begin{array}{l}\mathrm{Di} \text { et al., } \\
2003\end{array}$ \\
\hline $\begin{array}{l}\text { Increased } \\
\text { flavonol } \\
\text { content }\end{array}$ & potato & Chi & $\begin{array}{l}\text { tuber-specific } \\
\text { overexpression }\end{array}$ & 2004 & $\begin{array}{l}\text { Lukaszewicz } \\
\text { et al., } 2004\end{array}$ \\
\hline $\begin{array}{l}\text { Heat-stable } \\
\text { vegetable oil }\end{array}$ & soybean & Fad3 & $\begin{array}{l}\text { seed-specific } \\
\text { silencing }\end{array}$ & 2004 & $\begin{array}{l}\text { Fillatti et al., } \\
2004\end{array}$ \\
\hline
\end{tabular}




\begin{tabular}{|c|c|c|c|c|c|}
\hline $\begin{array}{l}\text { Bruise } \\
\text { tolerance }\end{array}$ & potato & Ppo & $\begin{array}{l}\text { tuber-specific } \\
\text { silencing }\end{array}$ & 2004 & $\begin{array}{l}\text { Rommens et } \\
\text { al., } 2004\end{array}$ \\
\hline $\begin{array}{l}\text { Increased } \\
\text { carotenoid } \\
\text { and flavonoid } \\
\text { content }\end{array}$ & tomato & Det1 & $\begin{array}{l}\text { fruit-specific } \\
\text { silencing }\end{array}$ & 2005 & $\begin{array}{l}\text { Davuluri et } \\
\text { al., } 2005\end{array}$ \\
\hline $\begin{array}{l}\text { Reduced } \\
\text { allergen } \\
\text { content }\end{array}$ & apple & Mal d 1 & $\begin{array}{l}\text { constitutive } \\
\text { silencing }^{\mathrm{d}}\end{array}$ & 2005 & $\begin{array}{l}\text { Gilissen } \\
\text { al., } 2005\end{array}$ \\
\hline $\begin{array}{l}\text { Reduced } \\
\text { allergen } \\
\text { content }\end{array}$ & peanut & Ara h 2 & $\begin{array}{l}\text { constitutive } \\
\text { silencing }^{\mathrm{d}}\end{array}$ & 2005 & $\begin{array}{l}\text { Dodo et al., } \\
2005\end{array}$ \\
\hline $\begin{array}{l}\text { Extended shelf } \\
\text { life }\end{array}$ & tomato & Dhs & $\begin{array}{l}\text { constitutive } \\
\text { silencing }^{\mathrm{b}}\end{array}$ & 2005 & $\begin{array}{l}\text { Wang et al., } \\
2005\end{array}$ \\
\hline $\begin{array}{l}\text { Increased â- } \\
\text { carotene } \\
\text { content }\end{array}$ & potato & Lcy-e & $\begin{array}{l}\text { tuber-specific } \\
\text { silencing }\end{array}$ & 2006 & $\begin{array}{l}\text { Diretto } \\
\text { al., } 2006\end{array}$ \\
\hline $\begin{array}{l}\text { Enhanced } \\
\text { aroma }\end{array}$ & tomato & Aadc1A & $\begin{array}{l}\text { constitutive } \\
\text { overexpression }^{\mathrm{b}}\end{array}$ & 2006 & $\begin{array}{l}\text { Tieman } \\
\text { al., } 2006\end{array}$ \\
\hline $\begin{array}{l}\text { Enhanced } \\
\text { flavor }\end{array}$ & potato & $\begin{array}{l}\mathrm{R} 1 \\
+ \\
\mathrm{PhL}\end{array}$ & $\begin{array}{l}\text { tuber-specific } \\
\text { silencing }\end{array}$ & 2006 & $\begin{array}{l}\text { Rommens et } \\
\text { al., } 2006\end{array}$ \\
\hline $\begin{array}{l}\text { Reduced heat- } \\
\text { induced } \\
\text { acrylamide } \\
\text { content }\end{array}$ & potato & $\begin{array}{l}\mathrm{R} 1 \\
+ \\
\mathrm{PhL}\end{array}$ & $\begin{array}{l}\text { tuber-specific } \\
\text { silencing }\end{array}$ & 2006 & $\begin{array}{l}\text { Rommens et } \\
\text { al., } 2006\end{array}$ \\
\hline $\begin{array}{l}\text { Reduced } \\
\text { lignin content }\end{array}$ & alfalfa/feed & $\mathrm{C} 3 \mathrm{H}$ & $\begin{array}{l}\text { silencing in vascular } \\
\text { tissues }\end{array}$ & 2006 & $\begin{array}{l}\text { Ralph et al., } \\
2006\end{array}$ \\
\hline $\begin{array}{l}\text { Reduced } \\
\text { allergen } \\
\text { content }\end{array}$ & tomato & $\begin{array}{l}\text { Ltpg1 } \\
\text { Ltpg2 }\end{array}$ & $\begin{array}{l}\text { constitutive } \\
\text { silencing }^{\mathrm{d}}\end{array}$ & 2006 & $\begin{array}{l}\text { Le et al., } \\
2006\end{array}$ \\
\hline $\begin{array}{l}\text { Increased } \\
\text { folate content }\end{array}$ & tomato & Acds & $\begin{array}{l}\text { fruit-specific } \\
\text { overexpression }\end{array}$ & 2007 & $\begin{array}{l}\text { Diaz de la } \\
\text { Garza et al., } \\
2007\end{array}$ \\
\hline $\begin{array}{l}\text { Reduced heat- } \\
\text { induced } \\
\text { acrylamide } \\
\text { content }\end{array}$ & potato & Apg1 & $\begin{array}{l}\text { tuber-specific } \\
\text { overexpression }\end{array}$ & 2007 & $\begin{array}{l}\text { Rommens, } \\
2007\end{array}$ \\
\hline $\begin{array}{l}\text { Reduced heat- } \\
\text { induced } \\
\text { acrylamide } \\
\text { content }\end{array}$ & potato & $\begin{array}{l}\text { Asn1 } \\
+ \\
\text { Asn2 }\end{array}$ & $\begin{array}{l}\text { tuber-specific } \\
\text { silencing }\end{array}$ & 2007 & $\begin{array}{l}\text { Rommens, } \\
2007\end{array}$ \\
\hline
\end{tabular}

${ }^{a}$ Concept demonstrated inArabidopsis. ${ }^{b}$ Molecular strategies may be improved upon by employing tissue-specific promoters. ${ }^{\mathrm{c}}$ Gene isolated from Arabidopsis. ${ }^{\mathrm{d}}$ Multigene silencing constructs may be used to simultaneously inactivate various allergen-encoding genes. 
Table.2 Intragenic crops developed or currently under development

\begin{tabular}{|c|c|c|c|c|c|}
\hline Crop & Type & Prom./term./spacer from & Gene & Trait & $\begin{array}{l}\text { Referen } \\
\text { ce }\end{array}$ \\
\hline Potato & Silencing & $\begin{array}{l}\text { GBSS/nos } \\
\text { Spacer: GBSS-fragment } \\
\text { GBSS/GBSS } \\
\text { Spacer: GBSS-fragment }\end{array}$ & GBSS & $\begin{array}{l}\text { High } \\
\text { amylopectin }\end{array}$ & $\begin{array}{l}\text { de } \\
\text { Vetten } \text { et } \\
\text { al., } 2003\end{array}$ \\
\hline Potato & Silencing & $\begin{array}{l}\text { GBSS/Ubi3 } \\
\text { Spacer: Ubi7-fragment }\end{array}$ & Ppo & $\begin{array}{l}\text { Preventing } \\
\text { black spot } \\
\text { bruise }\end{array}$ & $\begin{array}{l}\text { Rommen } \\
\text { s et al., } \\
2004\end{array}$ \\
\hline Potato & Silencing & $\begin{array}{l}\text { GBSS/Ubi3 } \\
\text { Spacer: Ubi7-fragment }\end{array}$ & $\begin{array}{l}\text { Ppo, } \\
\text { R1, } \\
\text { PhL }\end{array}$ & $\begin{array}{l}\text { Preventing } \\
\text { black spot } \\
\text { bruise. } \\
\text { Limiting cold- } \\
\text { induced } \\
\text { degradation }\end{array}$ & $\begin{array}{l}\text { Rommen } \\
\text { s et al., } \\
2006\end{array}$ \\
\hline Potato & Silencing & $\begin{array}{l}\text { Prom.Agp/Prom.GBS* } \\
\text { Spacer: Ubi7-fragment }\end{array}$ & $\begin{array}{l}\text { StAs1,S } \\
\text { tAS2 }\end{array}$ & $\begin{array}{l}\text { Limit } \\
\text { acrylamide in } \\
\text { French Fries }\end{array}$ & $\begin{array}{l}\text { Rommen } \\
\mathrm{s} \text { et al., } \\
2008\end{array}$ \\
\hline Potato & Silencing & $\begin{array}{l}\text { Prom.Agp/Prom.GBS* } \\
\text { Spacer: Ubi7-fragment }\end{array}$ & StAs1 & $\begin{array}{l}\text { Limit } \\
\text { acrylamide in } \\
\text { French Fries }\end{array}$ & $\begin{array}{l}\text { Chawla } \\
\text { et al., } \\
2012\end{array}$ \\
\hline Apple & Expression & $\mathrm{RbcS} / \mathrm{RbcS}$ & HcrVf2 & $\begin{array}{l}\text { Scab } \\
\text { resistance }\end{array}$ & $\begin{array}{l}\text { Joshi et } \\
\text { al., } 2011\end{array}$ \\
\hline Strawberry & $\begin{array}{l}\text { Overexpress } \\
\text { ion }\end{array}$ & FaExp2/FaExp2 & PGIP & $\begin{array}{l}\text { Gray mould } \\
\text { resistance }\end{array}$ & $\begin{array}{l}\text { Schaart, } \\
2004\end{array}$ \\
\hline Alfalfa & Silencing & $\begin{array}{l}\text { PromPetE/PromPetE* } \\
\text { Spacer: Comt-fragment }\end{array}$ & Comt & $\begin{array}{l}\text { Reduced } \\
\text { levels } \\
\text { lignin }\end{array}$ & $\begin{array}{l}\text { Weeks et } \\
\text { al., } 2008\end{array}$ \\
\hline $\begin{array}{l}\text { Perennial } \\
\text { ryegrass }\end{array}$ & $\begin{array}{l}\text { Overexpress } \\
\text { ion }\end{array}$ & n.m. but from species itself & Lpvp1 & $\begin{array}{l}\text { Drought } \\
\text { tolerance }\end{array}$ & $\begin{array}{l}\text { Bajaj et } \\
\text { al., } 2008\end{array}$ \\
\hline
\end{tabular}

*This type of 'convergent transcription' silencing construct with two promoters was shown to be very efficient (Yan et al., 2006). n.m.: not mentioned

Present status of intragenic crops' regulation

The simplicity, timeframe and cost of endorsement of the intragenic crops are under development will be dependent upon the future regulations of these crops. In the majority of the countries, the release of intragenic crops falls currently under the regulatory guidelines as similar as the crops that are transgenic.

\section{Limitations of intragenics}

Although the intragenic technology is enlightening considerable advantages more than the transgenic counterpart, conversely, still there are a petite number of limitations 
present related to this novel tool. One of the chief disadvantages contributed by the intragenic approach compared to transgenic, is that characters that are outside to the sexually well-matched gene pool cannot be introduced. In addition, extraordinary proficiency and time are compulsory for successful development of intragenic crops as compared to the transgenic crops.

The application of intragenic practice boosts the opportunity to introgress the ideal favoured genes into the fresh cultivars, without troubling their constructive characteristics. Conventional methods in plant breeding rely on random modifications of genome and are not easy to apply to either eradicate undesirable features or activate the dormant traits. Moreover, improvements of definite characteristics could be coupled with inadvertent as well as unnoticed changes in genetic level that compromise quality of food. These issues are addressed efficiently by accurately recombining native elements in vitro and introducing the resulting expression cassettes into plants using marker-free and all-native DNA transformation. An evaluation of these low-risk crops should be focused on any potential safety issues to the consumer. We propose to lessen the regulatory yoke for intragenic crops as compared to transgenic crops.

\section{References}

Agius F, González-Lamothe R, Caballero JL, Muñoz-Blanco J, Botella MA, Valpuesta $\mathrm{V}$. Engineering increased vitamin $\mathrm{C}$ levels in plants by overexpression of a Dgalacturonic acid reductase. Nature biotechnology. 2003 Feb; 21(2):177-81.

Akeley RV, Mills WR, Cunningham CE, Watts J. Lenape: a new potato variety high in solids and chipping quality. American Potato Journal. 1968 Apr 1;45(4):142-5.

Ames BN, Profet M, Gold LS. Nature's chemicals and synthetic chemicals: comparative toxicology. Proceedings of the National Academy of Sciences. 1990 Oct 1;87(19):7782-6.

Bajaj S, Puthigae S, Templeton K, Bryant C, Gill G, Lomba P, Zhang H, Altpeter F, Hanley Z. Towards engineering drought tolerance in perennial ryegrass using its own genome. In6th Canadian plant genomics workshop 2008 Jun 23 (Vol. 62).

Bauer MW, Bauer MW, Gaskell G, Durant J, editors. Biotechnology-the making of a global controversy. Cambridge University Press; 2002 Sep 5.

Bradford KJ, Van Deynze A, Gutterson N, Parrott W, Strauss SH. Regulating transgenic crops sensibly: lessons from plant breeding, biotechnology and genomics. Nature biotechnology. 2005 Apr; 23(4):439-44.

Chawla R, Shakya R, Rommens CM. Tuber- specific silencing of asparagine synthetase- 1 reduces the acrylamide- forming potential of potatoes grown in the field without affecting tuber shape and yield. Plant biotechnology journal. 2012 Oct;10(8):913-24.

Davuluri GR, Van Tuinen A, Fraser PD, Manfredonia A, Newman R, Burgess D, Brummell DA, King SR, Palys J, Uhlig J, Bramley PM. Fruit-specific RNAimediated suppression of DET1 enhances carotenoid and flavonoid content in tomatoes. Nature biotechnology. 2005 Jul;23(7):890-5.

de la Garza RD, Quinlivan EP, Klaus SM, Basset GJ, Gregory JF, Hanson AD. Folate biofortification in tomatoes by engineering the pteridine branch of folate synthesis. Proceedings of the National Academy of Sciences. 2004 Sep 21;101(38):13720-5.

de Vetten N, Wolters AM, Raemakers K, van der Meer I, ter Stege R, Heeres E, Heeres $\mathrm{P}$, Visser R. A transformation method for obtaining marker-free plants of a crosspollinating and vegetatively propagated crop. Nature biotechnology. 2003 Apr; 21(4):439-42. 
Devi EL, Chongtham SK, Holeyachi P, Kousar N, Singh M, Behera C, Telem RS, Singh NA, Wani SH. Cisgenesis and intragenesis: twin sisters for crop improvement. Res J Agric For Sci. 2013; 1:22-6.

Dharmapuri S, Rosati C, Pallara P, Aquilani R, Bouvier F, Camara B, Giuliano G. Metabolic engineering of xanthophyll content in tomato fruits. Febs Letters. 2002 May 22;519(1-3):30-4.

Di R, Kim J, Martin MN, Leustek T, Jhoo J, Ho CT, Tumer NE. Enhancement of the primary flavor compound methional in potato by increasing the level of soluble methionine. Journal of agricultural and food chemistry. $2003 \quad$ Sep 10;51(19):5695-702.

Diretto G, Tavazza R, Welsch R, Pizzichini D, Mourgues F, Papacchioli V, Beyer P, Giuliano G. Metabolic engineering of potato tuber carotenoids through tuberspecific silencing of lycopene epsilon cyclase. BMC plant biology. 2006 Dec 1;6(1):13.

Dodo $\mathrm{H}$, Konan $\mathrm{K}$, Viquez $\mathrm{O}$. A genetic engineering strategy to eliminate peanut allergy. Current allergy and asthma reports. 2005 Jan 1;5(1):67.

Gaskell G, Bauer MW, editors. Biotechnology 1996-2000: The years of controversy. London: Science Museum; 2001.

Gendel SM, Jenkins JA. Allergen sequence databases. Molecular nutrition \& food research. 2006 Jul;50(7):633-7.

Gilissen LJ, Bolhaar ST, Matos CI, Rouwendal GJ, Boone MJ, Krens FA, Zuidmeer L, van Leeuwen A, Akkerdaas J, HoffmannSommergruber K, Knulst AC. Silencing the major apple allergen Mal d 1 by using the RNA interference approach. Journal of allergy and clinical immunology. 2005 Feb 1; 115(2): 364-9.

Harrison MS, Wehbi M, Obideen K. Celiac disease: more common than you think. Cleveland Clinic journal of medicine. 2007 Mar 1; 74(3): 209.

Herman EM, Helm RM, Jung R, Kinney AJ. Genetic modification removes an immunodominant allergen from soybean. Plant physiology. 2003 May 1;132(1):3643.

Holme IB, Wendt T, Holm PB. Intragenesis and cisgenesis as alternatives to transgenic crop development. Plant Biotechnology Journal. 2013 May; 11(4):395-407.

Jacobsen E, Schouten HJ. Cisgenesis strongly improves introgression breeding and induced translocation breeding of plants. Trends in biotechnology. 2007 May 1;25(5):219-23.

Joshi SG, Schaart JG, Groenwold R, Jacobsen E, Schouten HJ, Krens FA. Functional analysis and expression profiling of HcrVf1 and HcrVf2 for development of scab resistant cisgenic and intragenic apples. Plant molecular biology. 2011 Apr 1;75(6):579-91.

Kaeppler HF. Food safety assessment of genetically modified crops. Agronomy Journal. 2000 Jul 1;92(4):793-7.

Kopisch-Obuch FJ, McBroom RL, Diers BW. Association between soybean cyst nematode resistance loci and yield in soybean. Crop science. 2005 May 1;45(3):956-65.

Lassen J, Madsen KH, Sandøe P. Ethics and genetic engineering-lessons to be learned from GM foods. Bioprocess and Biosystems Engineering. 2002 Jan 1; 24(5): 263-71.

Le LQ, Lorenz Y, Scheurer S, Fötisch K, Enrique E, Bartra J, Biemelt S, Vieths S, Sonnewald U. Design of tomato fruits with reduced allergenicity by dsRNAi- mediated inhibition of ns- LTP (Lyc e 3) expression. Plant biotechnology journal. 2006 Mar;4(2):231-42.

Liu C, Tian Y, Shen Q, Jiang H, Ju R, Yan T, Liu C, Mang $K$. Cloning of 1aminocyclopropane-1-carboxylate (ACC) synthetase cDNA and the inhibition of fruit ripening by its antisense RNA in transgenic tomato plants. Chinese journal of biotechnology. 1998; 14(2): 75.

Liu Q, Singh SP, Green AG. High-stearic and high-oleic cottonseed oils produced by hairpin RNA-mediated post- 
transcriptional gene silencing. Plant physiology. 2002 Aug 1; 129(4): 173243.

Lukaszewicz M, Matysiak-Kata I, Skala J, Fecka I, Cisowski W, Szopa J. Antioxidant capacity manipulation in transgenic potato tuber by changes in phenolic compounds content. Journal of Agricultural and Food Chemistry. 2004 Mar 24; 52(6): 1526-33.

Mathews H, Clendennen SK, Caldwell CG, Liu XL, Connors K, Matheis N, Schuster DK, Menasco DJ, Wagoner W, Lightner J, Wagner DR. Activation tagging in tomato identifies a transcriptional regulator of anthocyanin biosynthesis, modification, and transport. The Plant Cell. 2003 Aug 1; 15(8): 1689-703.

Moradpour M, Abdullah SN. Cisgenesis and Intragenesis as new strategies for crop improvement. In: Crop Improvement 2017 (pp. 191-216). Springer, Cham.

Oeller PW, Lu MW, Taylor LP, Pike DA, Theologis A. Reversible inhibition of tomato fruit senescence by antisense RNA. Science. 1991 Oct 18; 254(5030): 437-9.

Ralph J, Akiyama T, Kim H, Lu F, Schatz PF, Marita JM, Ralph SA, Reddy MS, Chen F, Dixon RA. Effects of coumarate 3hydroxylase down-regulation on lignin structure. Journal of Biological Chemistry. 2006 Mar 31; 281(13): 884353.

Römer S, Lübeck J, Kauder F, Steiger S, Adomat C, Sandmann G. Genetic engineering of a zeaxanthin-rich potato by antisense inactivation and cosuppression of carotenoid epoxidation. Metabolic engineering. 2002 Oct 1;4(4):263-72.

Rommens CM, Bougri O, Yan H. Rommens CM, Humara JM, Ye J., Yan H., Richael C., Zhang L., Perry R., and Swords K. Crop Improvement through Modification of the Plant's Own Genome. This article has been retracted at the request of the authors. Retraction is based on three inaccurate statements of facts that are associated with a plant-derived transfer DNA. Two of the inaccuracies were described on p. 422.

Rommens CM, Haring MA, Swords K, Davies HV, Belknap WR. The intragenic approach as a new extension to traditional plant breeding. Trends in Plant Science. 2007 Sep 1; 12(9): 397-403.

Rommens CM, Humara JM, Ye J, Yan H, Richael C, Zhang L, Perry R, Swords K. Crop improvement through modification of the plant's own genome. Plant Physiology. 2004 May 1; 135(1): 421-31.

Rommens CM, Yan H, Swords K, Richael C, Ye J. Low- acrylamide French fries and potato chips. Plant biotechnology journal. 2008 Oct; 6(8): 843-53.

Rommens CM, Ye J, Richael C, Swords K. Improving potato storage and processing characteristics through all-native DNA transformation. Journal of Agricultural and Food Chemistry. 2006 Dec 27; 54(26): 9882-7.

Rommens CM. All-native DNA transformation: a new approach to plant genetic engineering. Trends in Plant Science. 2004 Sep 1; 9(9): 457-64.

Rommens CM. Intragenic crop improvement: Combining the benefits of traditional breeding and genetic engineering. Journal of agricultural and food chemistry. 2007 May 30; 55(11): 4281-8.

Rommens CM. Intragenic crop improvement: Combining the benefits of traditional breeding and genetic engineering. Journal of agricultural and food chemistry. 2007 May 30; 55(11):4281-8.

Schaart JG, Visser RG. Novel plant breeding techniques. Consequences of new genetic modification-based plant breeding techniques in comparison to conventional plant breeding. Cogem; 2009.

Schaart JG. Towards consumer-friendly cisgenic strawberries which are less susceptible to Botrytis cinerea. 2004.

Schouten HJ, Krens FA, and Jacobsen E. Cisgenic plants are similar to traditionally bred plants. EMBO Rep. 2006; 7: 750753. 
Schwall GP, Safford R, Westcott RJ, Jeffcoat R, Tayal A, Shi YC, Gidley MJ, Jobling SA. Production of very-high-amylose potato starch by inhibition of SBE A and B. Nature biotechnology. 2000 May; 18(5): 551-4.

Sheehy RE, Kramer M, Hiatt WR. Reduction of polygalacturonase activity in tomato fruit by antisense RNA. Proceedings of the National Academy of Sciences. 1988 Dec 1; 85(23): 8805-9.

Shintani D, DellaPenna D. Elevating the vitamin $\mathrm{E}$ content of plants through metabolic engineering. Science. $1998 \mathrm{Dec}$ 11; 282(5396): 2098-100.

Song J, Bradeen JM, Naess SK, Raasch JA, Wielgus SM, Haberlach GT, Liu J, Kuang H, Austin-Phillips S, Buell CR, Helgeson JP. Gene RB cloned from Solanum bulbocastanum confers broad spectrum resistance to potato late blight. Proceedings of the national academy of sciences. 2003 Aug 5; 100(16): 9128-33.

Tareke E, Rydberg P, Karlsson P, Eriksson S, Törnqvist M. Analysis of acrylamide, a carcinogen formed in heated foodstuffs. Journal of agricultural and food chemistry. 2002 Aug 14; 50(17): 49985006.

Tieman D, Taylor M, Schauer N, Fernie AR, Hanson AD, Klee HJ. Tomato aromatic amino acid decarboxylases participate in synthesis of the flavor volatiles 2phenylethanol and 2-phenylacetaldehyde. Proceedings of the National Academy of Sciences. 2006 May 23; 103(21): 828792.

Van Eenennaam AL, Lincoln K, Durrett TP, Valentin HE, Shewmaker CK, Thorne GM, Jiang J, Baszis SR, Levering CK, Aasen ED, Hao M. Engineering vitamin E content: from Arabidopsis mutant to soy oil. The Plant Cell. 2003 Dec 1;15(12):3007-19.

Wang TW, Zhang CG, Wu W, Nowack LM, Madey E, Thompson JE. Antisense suppression of deoxyhypusine synthase in tomato delays fruit softening and alters growth and development. Plant physiology. $2005 \mathrm{Jul}$ 1;138(3):1372-82.

Weeks JT, Ye J, Rommens CM. Development of an in planta method for transformation of alfalfa (Medicago sativa). Transgenic research. 2008 Aug 1; 17(4): 587-97.

Yan H, Chretien R, Ye J, Rommens CM. New construct approaches for efficient gene silencing in plants. Plant physiology. 2006 Aug 1; 141(4): 1508-18.

Zitnak A, Johnston GR. Glycoalkaloid content of B5141-6 potatoes. American Potato Journal. 1970 Jul 1; 47(7):2 56-60.

\section{How to cite this article:}

Mainak Barman, Saipayan Ghosh, Kingsuk Das and Sabir Ahmed Mondol. 2020. Intragenesis as a Sustainable Crop Improvement Method: A Review. Int.J.Curr.Microbiol.App.Sci. 9(04): 773-782. doi: https://doi.org/10.20546/ijcmas.2020.904.092 